Evolutionary Ecology, vol. 8, no. 3, 1994, pp. 299-314

\title{
Spatial analysis of vector-borne disease: a four-species model
}

\author{
Boleslaw Szymanski \\ Department of Computer Science \\ Rensselaer Polytechnic Institute \\ Troy, NY 12180
}

\author{
Tom Caraco \\ Department of Biological Sciences \\ SUNY Albany \\ Albany, NY 12192
}

\section{Summary}

Disease can influence a host population's dynamics directly or indirectly through effects on the host's interactions with competitors and exploiters. We present a stochastic, spatially explicit model for the epidemiological landscape of a vector-borne disease. Two host species, of unequal competitive strength, are attacked by a selective parasite; the parasite serves as a vector for a pathogen. We emphasize the importance of the ecological stencil, the local area where ecological interactions govern a site's species composition. We point out how parallel computing can efficiently employ the geometry of the stencil's local transitions to predict large-scale spatio-temporal patterns of the model community.

\section{Introduction}

Spatial heterogeneity in abiotic factors and biotic processes often generates patterns in population dynamics and the resulting attributes of ecological communities. Despite a long-standing recognition of the significance of spatial variation, analytical and computational models of spatially explicit, multispecies interactions have only recently influenced community theory (e.g. Hastings, 1990; Kareiva, 1990). We develop a spatially explicit model for the epidemiological landscape of a vector-borne disease. We focus on the "ecological stencil", the local area where ecological interactions govern the species composition at any specific location. Simulation of the local transition probabilities defining the stencil will predict large-scale spatio-temporal patterns of the model community.

We first mention a few examples of vector-borne disease. Then we present a model for the dispersal-mortality dynamics of two competing host species, a pathogen that infects the hosts, and a parasite (on the hosts) that serves as a vector for the pathogen. Using a simplified version of the model, we show how variation in the size of the ecological stencil can influence "quasi-stationary" abundances of species competing in a probabilistic density-dependent manner. We briefly comment on the computational requirements for implementing the model efficiently (e.g. Sinharoy and Szymanski, 1993). We outline a series of applications of the model; these include spatially explicit analyses of ecological dispersal, the spread of an epidemic, and cultural diffusion of information. Finally, we consider how our model might provide spatial representations of evolutionary processes.

\section{Vector-borne disease}

Pathogens can influence a host's birth and death rates directly (e.g. Anderson, 1991), or indirectly through effects on the host's interactions with competitors and exploiters (e.g. Hochberg et al., 1990; Schall, in press). Ecological models of epidemics generally assume that a pathogen is directly transmitted when an infective host contacts a susceptible host individual. Recently, however, more attention has been directed to vector-borne diseases where individual parasites exploit many host individuals and consequently can transport a pathogen 
from an infected to an uninfected host. If a parasite/vector exploits hosts in an economically selective manner, foraging theory (e.g. Stephens and Krebs, 1986) may help predict spatial characteristics of epidemics.

To motivate our model, we briefly mention a few examples of vector-borne diseases that may influence community structure:

1. Power $(1987,1989)$ discusses herbivorous insects that transmit viral pathogens between host plants. In one case insects carry a virus that attacks both wild and cultivated grasses; insect host preferences consequently affect the frequency of infection in the Hxfferent host species.

2. Dodder (Cuscuta spp.) is a clonal plant that parasitizes other plants. In North America most dodder species exploit either herbaceous plants or small shrubs. Hosford (1967) reviewed the literature on pathogen transmission through dodder's vascular system and listed 56 viruses. Kelly (in press) demonstrates that dodder can discriminate between nitrogen-poor and nitrogen-rich host plants; a dodder stem will avoid the former to parasitize the latter. Consequent effects on the spatial propagation of disease are plausible.

3. Schall (in press) has studied saurian malaria in two Anolis species on the island of St. Maarten. The two lizards, A. wattsi and A. gingivinus, are similar in size and compete ecologically (Roughgarden et al., 1984). However, A. gingivinus appears the stronger competitor; it occurs throughout the island. A. wattsi has a restricted distribution. The malarial agent Plasmodium azurophilum seldom infects A. wattsi, but is common in A. gingivinus. Where malaria occurs, both lizards can be found. But in areas where malaria is absent (dry habitats), only the superior competitor occurs. Schall (in press) hypothesizes that malaria mediates the outcome of interspecific competition between lizards. Coexistence may be possible only in habitats that include the biting-insect vector of malaria. None of these examples of vector-borne disease, and only a few direct-contact epidemics, have been modeled as explicit dynamical processes in a two dimensional space (see Bartholomew, 1983; Murray et al., 1986).

\section{The ecological stencil}

Consider an environment $\mathrm{E}$ consisting of a finite number $\mathrm{J}$ of sites. The vector $N_{t}=\left[n_{1} n_{2} \ldots n_{J}\right]^{T}$ describes the state of the environment at time $t: n_{k} \in \xi_{k}$ is the value of the attribute that characterizes site $k$, and $\xi_{k}$ is a finite set of all possible values of this attribute. In the proposed model $n_{k}$ reveals the identity of the species occurring at each site $k$. The initial condition of the environment $E$ is described by the vector $N_{0}$. The state space $S$ of the vectors $N_{t}$ is defined as $S=\xi_{1} \times \xi 2 \ldots \times \xi_{J}$. The function $\pi: S \mapsto[0,1]$ is a random field if at every time $t$, The random field is a distribution assigning state probabilities. The properties of $\pi\left(N_{t}\right)$ at any time $t$, as well as at stochastic equilibrium (assuming an equilibrium exists), will necessarily depend on the transition probabilities between the elements of $S$.

Site $k$ is surrounded by neighboring sites $\delta k ; \delta k \subset(E-\{k\})$. Let $N_{t} \delta k$ be a subvector of $N_{t}$ that consists of values of all attributes characterizing the sites in $\delta k$ at time $t$. We refer to $\delta k$ as the ecological stencil for site $k$, implying that the state of the sites in $\delta k$, as well as the state at site $k$ itself, govern the probabilities of transition among the states $\xi_{k}$. That is, the abiotic and biotic processes driving the stochastic variation in $n_{k}$ will be described by a function of both $n_{k}$ and the state subvector $N_{t} \delta k$.

Let $\operatorname{Pr}\left[n_{k}=j i, N_{t}(E-\{k\})\right]$ denote the probability that site $k$ is in state $j$ at time $(t+\Delta t)$, given that site $k$ was in state $i$, and the rest of the environment was in state $N E-k$ at time $t . \operatorname{Pr}\left[n_{k}=j i, N_{t}(E-\{k\})\right]$ is the one-step transition probability for a change in the species composition at site $k$. In general, change at any particular site may depend on the entire vector $N_{t}$, i.e. on the state of the entire environment. But such strong interdependence among sites seldom will be reasonable biologically. Imposing our ecological stencil, we have

$$
\operatorname{Pr}\left[n k=j i, N_{t}(E-k)\right]=\operatorname{Pr}\left[n k=j i, N_{t} \delta k\right]
$$

The stochastic dynamics of the species composition at any site $\mathrm{k}$ will depend only on the state at this site and the state of the ecological stencil. The ecological stencil $\delta k$ is, in a sense, a geometrical generalization of the "neighborhood effect" analyzed in some recent models for the population dynamics of annual plants (e.g. Pacala and Silander 1985). The size and shape of $\delta k$ may vary with abiotic factors. For example, the strength and 
direction of prevailing winds may imply that only particular sites can disperse propagules that could become established at a given site. Biotic processes will also influence the ecological stencil. For vector-borne disease dispersal capacities of hosts and parasites, in both absolute and relative senses, may regulate their interaction and, consequently, the effect of a pathogen on hosts.

\section{Parameters of the model}

Our objective is understanding how the local, probabilistic dynamics of the ecological stencil can influence large-scale spatio-temporal patterns in an ecological community. Our model assumes a four-species community. Two host species are exploited by a single parasitic species. We identify the host species by a binary variable $h \in 0,1$. $h=1$ implies the "good" host (from the perspective of the selective parasite), and $h=0$ implies the "bad" host. The parasite can expect to persist longer and infest new hosts more quickly when it exploits a good host. The parasite acts as a vector, transmitting a pathogen from infected hosts to uninfected hosts. The ecosystem is assumed to be closed to immigration, so that extinction of one or more species is possible. The environment contains $J$ sites arrayed as a rectangular lattice. Each site $k$ is in one of nine possible states, hence $\xi_{k}=1, \ldots, 9$. A particular site may be empty (state 1), or may be occupied by one of the host species, but not both. If a host h occupies site $k$, it may occur (i) alone: state $3-h$, (ii) with the parasite: state $5-h$, (iii) with the pathogen: state $7-h$, or (iv) with both the parasite and the pathogen: state $9-h$. Table 1 summarizes the designation of the elements of $\xi_{k}$. Of course, $\xi_{k}$ can be collapsed to represent simpler interactions. For example,

\begin{tabular}{|c|c|c|c|}
\hline state & host & parasite & pathogen \\
\hline \hline 1 & absent & absent & absent \\
\hline $3-h$ & $h$ & absent & absent \\
\hline $5-h$ & $h$ & present & absent \\
\hline $7-h$ & $h$ & absent & present \\
\hline $9-h$ & $h$ & present & present \\
\hline
\end{tabular}

Table 1: Designation of the state space for each site $\mathrm{k}$.

$\xi_{k}=1,2,3$ when the two competing "host" species are not subject to either parasitism or disease. Let $p_{k}(i, j)$ represent the one-step transition probability from state $i$ to state $j$. To define $p_{k}(i, j)$ we need to consider all sites in $\delta k$, the ecological stencil for site $k$. Let $P_{i}$ denote the set of states to which transitions from state $i$ have positive probabilities: $P i=j \in \xi_{k} p_{k}(i, j)>0$. Since $\sum_{j} p_{k}(i, j)=1$ for $j \in P_{i}$, the probability of the transition preserving the state can be expressed as:

The dispersal of a host species from site $l$ to some other site $k$ can be divided into three temporal stages: reaching site $k$ (assuming juveniles/propagules disperse), initiating development at this site, and achieving reproductive maturity by out-competing (the) other species there. For example, a host plant (the example we use) must reach site $k$ with a seed, the seed must germinate, and the plant must compete successfully for space before reproducing. Corresponding to the three stages, we define the following three probabilities:

1. $p_{h r}(l, k)$ is the probability that a host of type $h$ reaches from site $l$ to site $k$. This probability will ordinarily depend on the species composition at site $l$ and may also depend on the distance from $l$ to $k$. Simply to outline the logic of our model, assume initially that this probability is constant within the ecological stencil $\delta k$ and equals $p_{h r}$. If distance strongly governed dispersal, a more realistic model might assume that the probability of advancing from site $l$ to site $k$ decreases with each intermediate site traversed. Geometrically decaying dispersal offers a reasonable model for this decrease (e.g. [16]). That is, we could write $p_{h r}(l, k)=p_{h r} w^{k-l}$ for $w<1$. We assume that the good host has certain ecological advantages over the bad host, so that $p_{1 r} \geq p_{0 r}$.

2. $p_{h g}(k)$ is the probability of a type $h$ host individual germinating from a single propagule at site $k$. This probability will often be independent of the site, in which case we shall denote it phg. However, in diversified 
habitats this probability can depend on the site $k$. The ecological superiority of the good host noted above implies that $p_{1 g} \geq p_{0 g}$.

3. $p_{c}$ is the probability of a good host developing to reproductive maturity when both host types germinate at the same site. Since we assume the good host type has a competitive advantage, $p c>0.5$.

Under these assumptions the resulting probability $p_{h w}(k)$ of a type $h$ host successfully occupying an open site $k$ during a single time interval of length $\Delta t$ is: where $m_{h k}$ denotes the number of sites in the ecological stencil $\delta k$ that are populated by a host of type $h$. Applying the binomial formula twice, we find that:

Thus, assuming that (i) all sites in the ecological stencil occupied by a type $h$ host have the same probability $p_{h r}$ of reaching site $k$, and (ii) germination is independent of $k$, then the probability of an organism establishing itself at site $k$ is just dependent on the probability $p_{h e}=p_{h r} p_{h g}$. In the more realistic case where host dispersal to site $k$ depends on the presence/absence of the parasite and pathogen across the ecological stencil, the calculations are more complicated, but the logic is the same.

In our model direct competition following establishment takes place only between the two types of hosts. The impact of this competition on the state transition probabilities is discussed below. We use the term mortality to refer to changes in the state $n_{k}$ involving loss of one or more species at site $k$. Mortality implies death for a host individual, and termination of an infestation for the parasitic species. Pathogen mortality occurs when a host either recovers from infection or dies. That is, death of an exploited host is sufficient, but not necessary, for "mortality" of the parasitic and pathogenic species. If a species has mortality probability $\mu$, we assume that $\mu$ is constant in time. Hence the associated expected lifespan is finite and equals $\frac{1}{\mu}$. However, this assumption does not constrain the maximal feasible lifespan. A more sophisticated model could represent the mortality rate as $\mu^{(t)}$, defined as follows. Consider a cohort of sites (i.e. a set of sites entering the same state at $t=0$ ). Then still occupied at time $t$ where "mortality" occurs by time $(t+\Delta t)$. If $M$ is the maximal lifespan for the species, then: the organism, occupying a site that is both in the source state and lies within the ecological stencil $\delta k$, disperses and becomes established at site $k$ with probability $\rho_{h+j} ; j=2,4,6$. The same organism suffers mortality at the same site with probability $\mu_{h+j}$. Note that dispersal of the pathogen to site $\mathrm{k}$ requires that the vector (the parasite) be present at both the source and site $k$. For

convenience the table assumes the same time tick $\Delta t$ for the various transitions. Finally, if both the good and bad host species become established at site $k$, the good host prevails in interspecific competition and matures with probability $p_{c}>0.5$. Table 2 shows the notation for the dispersal and mortality parameters of the model.

\begin{tabular}{|c|c|c|c|c|}
\hline organism & site $k$ & $p_{h r} p_{h g}(k)$ & source state & $\mu$ \\
\hline \hline host $h$ & open & $\rho_{h}$ & $3-h$ & $\mu_{h}$ \\
\hline host $h$, parasitized & open & $\rho_{h+2}$ & $5-h$ & $\mu h+2$ \\
\hline host $h$, infected & open & $\rho_{h+4}$ & $7-h$ & $\mu_{h+4}$ \\
\hline host $h$, parasitized and infected & open & $\rho_{h+6}$ & $9-h$ & $\mu_{h+6}$ \\
\hline parasite on host $h$ & host $g$ & $\alpha_{h g}$ & $5-h \& 9-h$ & $\mu_{d}$ \\
\hline pathogen on host with parasite & host $h$ \& parasite & $\beta_{h}$ & $9-h$ & $\mu_{v}$ \\
\hline
\end{tabular}

Table 2: Parameters of the model

For simplicity we assume that the ecolgical stencils for both hosts and parasites are the same. However, a more computationally complex extension of our model would consider systems where the spatial pattern of host dispersal differs from the parasite's dispersal pattern. Another generalization of our model would allow different values of the simulation tick for hosts versus parasites and pathogens. Host dispersal (but not necessarily host death) could occur at a temporal scale that differs from the scale of parasite or pathogen attacks.

\section{Interspecific influences on parameters}

Now we let ecological interactions among the four species govern the relative values of the probabilistic transitions within the ecological stencil. For example, the probability that a host individual reaches site $k$ with a 
propagule $\left(p_{h r}\right)$ should depend on whether or not the host is exploited. To account for these effects, we let both the parasite and the pathogen reduce the dispersal and increase the mortality of each host species. Hence $\rho_{h+2}<\rho_{h}, \rho_{h+4}<\rho_{h}, \mu_{h}<\mu_{h+2}$, and $\mu_{h}<\mu_{h+4}$. Our initial simulations assume an additive effect of parasitism and infection; hence $\rho_{h+6}-\rho_{h+4}=\rho_{h+2}-\rho_{h}$, and $\mu_{h}-\mu_{h+2}=\mu_{h+4}-\mu_{h+6}$. However, some diseases may affect dispersal and mortality differently. Note that by setting $\rho_{h+6}=\rho_{h+4}=0$ we can model pathogenic disease that precludes reproduction by an infected host. Since we assume that the good host is more efficient in exploiting the environment, we set $\rho_{1} \geq \rho_{0}$ and $\mu_{0}>\mu_{1}$.

Our model allows us to ask if selectivity at the behavioral level can influence spatial and temporal patterns at the community level. Specifically, we expect the parasite's host choice to influence community dynamics, especially if hosts are highly susceptible to the pathogen that the parasite may carry. We let $\alpha_{h g}(g, h=0,1)$ represent the "attack rate" on an unparasitized type- $g$ host at site $k$ from each parasitized type- $h$ host in the ecological stencil $\delta k$. The difference in host quality implies that the parasite attacks a given host type more readily while exploiting a good host. Therefore, we assume that $\alpha_{11}>\alpha_{01}$, and $\alpha_{10}>\alpha_{00}$. Selectivity of the parasite implies that a good host (when available) is attacked more readily than a bad host by a parasite exploiting a given host type. That is, we assume $\alpha_{11}>\alpha_{10}$, and $\alpha_{01}>\alpha_{00}$. The strength of the parasite's selectivity increases with the differences $\left(\alpha_{11}-\alpha_{10}\right)$ and $\left(\alpha_{01}-\alpha_{00}\right)$. Economic constraints and objectives might imply that these differences are subject to optimizing selection, so that the behavioral ecology of host choice could influence the spread of disease and community diversity.

$\beta_{h}$ is the pathogen-susceptibility parameter for host species $h$. We make no assumption about the relationship between $\beta_{1}$ and $\beta_{0}$. Good hosts might sometimes be less susceptible to disease than are bad hosts (suggesting that $\beta_{0}>\beta_{1}$ ). But Schall (in press) reports an ecologically interesting example of saurian malaria where $\beta_{0} \approx 0<\beta_{1}$.

Below we combine our assumptions concerning dispersal, mortality, selectivity and susceptibility to write the transition probabilities for the ecological stencil, the local dynamics allowing computational analyses of the largescale spatio-temporal properties of the four-species system. But first we consider some general relationships linking the size of the ecological stencil, density-dependent competition between hosts, and quasi-stationary species abundances.

Extinction versus dynamic equilibrium Extinction of various species constitutes an absorbing barrier. Although extinction is the ultimate equilibrium configuration of the system, the time to reach absorption can grow very large for $J>>1$ (see e.g., [8]). Consequently, extinction need not be a serious problem for realistic dispersal/mortality probabilities. Furthermore, we can solve for positive, quasi-stationary equilibria (see Sharma et al. 1982) to explore some biological predictions of our model.

An interesting example of density dependent dynamics arises when we consider the two host species without the parasite or pathogen. Suppose the good host invades the environment at low density after the (competitively inferior) bad host has reached an equilibrium density. The bad host can remain common, in quasi-stationary equilibrium with a low density of good hosts, until chance events (i.e. reasons other than the presence of the good host) induce a drastic decline in the bad host's density. Only then is the (competitively superior) good host able to propagate macroscopically. This sort of effect requires sufficiently large ecological stencils. We plan extensive computer simulation to locate the boundary size of the ecological stencils where this effect can occur.

To demonstrate this effect, let $d_{h}$ represent the average density of host $h(h=0,1)$, i.e. the number of type $h$ host individuals divided by the number of sites $J$. Therefore, $\left(J \mu_{h} d_{h}\right)$ is the expected number of deaths per time unit for hosts of type $h$. On the other hand, $\left\{J p_{k}(1,3-h)(1-d 0-d 1)\right\}$ is the expected number of births per time unit for host $h$. If natality/dispersal just balances mortality in each species, an equilibrium is established. By assumption, $d 0>>d 1$; the bad host is commmon and the good host is rare. Then, even if $p_{c}>0.5, \mu_{0}>\mu_{1}$, and $p_{1 r} p_{1 g}>p_{0 r} p_{0 g}$, we still can have $\delta k d_{0}>\delta k d_{1}$, where $\delta k$ is the number of sites in the ecological stencil. As a result of the bad host's greater density, $p_{k}(1,3)>p_{k}(1,2)$; an open site is more likely to be colonized by the bad host than by the good. The bad host has a density-dependent dispersal advantage that counters its mortality disadvantage. The equilibrium is maintained until stochastic variation about the expected system behavior allows the good host to advance.

For a single species system the equlibrium density d can be easily found by equating the mortality rate with the rate at which open sites are occupied:

$$
J \mu d=J(1-d)\left[1-\left(1-p_{h e}\right)^{d} \delta^{k}\right]
$$


Then, if $a=\left(1-p_{h e}\right) \delta^{k}$, we obtain:

$$
\mu d /(1-d)=1-a^{d}
$$

Clearly, the right hand side of this equation is always less than unity, so that $d<[1 /(1+\mu)]$. Setting $d=$ $[1 /(1+\mu)]-x$, the last equation yields:

$$
x a^{x} /(\mu+x+x \mu)=\left[a^{1 /(1+\mu)}\right] /(1+\mu)^{2}
$$

This equation shows how the interplay between value of $\mu, p_{h e}$, and the size of the ecological stencil $\delta k$ (the latter two define the value of a) influences $x$ and consequently governs the equilibrium density $d$. In the more interesting case of two competing species, the equations are more involved:

$$
\begin{aligned}
d_{1} \mu_{1} /\left(1-d_{0}-d_{1}\right) & =p_{1 w}\left[\left[1-p_{0 w}\left(1-p_{c}\right)\right]\right. \\
d_{0} \mu_{0} /\left(1-d_{0}-d_{1}\right) & =p_{0 w}\left(1-p_{1 w} p_{c}\right)
\end{aligned}
$$

where: If $d_{0}$ and $p_{0 e}$ are small (i.e., the density of the bad host and its probability of establishment in an open site are both low) then the bad host species will become extinct. However, for large stencils and sufficiently large bad host density, the probability $p_{0 w}$ approaches 1 . Hence, at equilibrium, we have: Since $p_{c}>0.5$ and $d_{0} \mu_{0}>>d_{1} \mu_{1}$, the value of $p_{1 w}$ may stay sufficiently small for the ecologically inferior bad host to remain common. When this effect occurs, $p_{1 w}$ is sufficiently small because the good host is rare, and low density across a large ecological stencil allows the bad host to acquire open sites often enough to maintain the (quasi-stationary) equilibrium.

This effect has some resemblance to pre-emptive competition reported in marine fouling commumities (Sutherland, 1974; Schoener 1976) and annual-plant communities (Bergelson 1990). However, in those cases adults of one generation (dead biomass in the latter case) interfered with larval settlement/seedling success. Our quasi-stationary equilibria are maintained through localized (i.e. within-stencil) numerical biases in a lottery competition between propagules for available space (see Shmida and Ellner, 1984).

\section{Transition probabilities for the four-species model}

When all four species are extant, the chance that a host of type $h$ will occupy an open site depends on the number of type- $h$ individuals in the ecological stencil about the site, and whether or not each of these individuals is infested by the parasite and/or infected by the pathogen. We retain the assumption that sites in $\delta k$ affect site $k$ independently.

Let $\sigma_{j} k$ represent the number of sites in the ecological stencil $\delta k$ that are in state $j\{j=1,2, \ldots 9\}$. The probability that a type- $h$ host establishes itself at site $k$ is: Since we assume good and bad hosts compete for space, the final probability of a type- $h$ host becoming established at site $k$ following dispersal and competition is: where $p_{c}>0.5$. Similarly, for parasitic attack on an uninfested type- $h$ host occupying site $k$, we find: If a type- $h$ host occupying site $k$ is infested by the parasite but has not acquired the disease, the parasite may act as a vector for the pathogen. The probability that this host acquires the disease is: Applying these relationships to the allowable transitions directs us to the dynamics of the full four-species model.

An empty site (state 1) may be colonized by a host of type $h$, or no change may occur. Hence the set of states that can be reached from state 1 with positive probability is $P_{1}=\{2,3,1\}$. When only the host of type $h$ occurs at site $k$ then the parasite may attack, the host may die, or no change may occur; hence $P_{3-h}=\{5-h, 1,3-h\}$.

When a site is occupied by a type- $h$ host and the parasite, the host may become free of the parasite, the host may die (so that the parasite no longer occupies the site), the parasite may transmit the pathogen to the host, or no change may occur. Therefore, $P_{5-h}=\{9-h, 3-h, 1,5-h\}$.

Consider site $k$ occupied by an infected host (of type $h$ ) without the parasite. The parasite may attack the host, the host may recover from the pathogen, or the host may die (leaving the site empty); hence $P_{7-h}=$ $\{9-h, 3-h, 1,7-h\}$. Recall that we assume the pathogen has no direct effect on the parasite's dispersal or selectivity.

If a site is occupied by a type- $h$ host that has been both attacked by the parasite and infected by the pathogen, then the host may become free of the parasite, the host may recover from the pathogen, or all three species may be lost in a single time interval. Consequently, $P_{9-h}=\{7-h, 5-h, 3-h, 1,9-h\}$. 


\begin{tabular}{|c|c|c|c|c|c|}
\hline from/to & 1 & $3-h$ & $5-h$ & $7-h$ & $9-h$ \\
\hline 1 & $p(1,1)$ & $p_{h w}$ & 0 & 0 & 0 \\
$3-h$ & $\mu_{h}$ & $p(3-h, 3-h)$ & $\left(1-\mu_{h}\right) p_{d s}$ & 0 & 0 \\
$5-h$ & $\mu_{h+2}$ & $\left(1-\mu_{h+2}\right) \mu_{d}$ & $p(5-h, 5-h)$ & 0 & $\left(1-\mu_{h+2}\right) p_{v s}\left(1-\mu_{d}\right)$ \\
$7-h$ & $\mu_{h+4}$ & $\left(1-\mu_{h+4}\right) \mu_{v}$ & 0 & $p(7-h, 7-h)$ & $\left(1-\mu_{h+4}\right) p_{d s}\left(1-\mu_{v}\right)$ \\
$9-h$ & $\mu_{h+6}$ & $\left(1-\mu_{h+6}\right) \mu_{d} \mu_{v}$ & $\left(1-\mu_{h+6}\right)\left(1-\mu_{v}\right) \mu_{d}$ & $\left(1-\mu_{h+6}\right)\left(1-\mu_{d}\right) \mu_{v}$ & $p(9-h, 9-h)$ \\
\hline
\end{tabular}

Table 3: Transition Probabilities

Table 3 shows the transition probabilities for the full four-species model of a vector-borne disease. The actual values at each site $k$ vary temporally, as the elements of the sub-vector $N_{t \delta k}$ change. Simulation requires that the transition probabilities be calculated for each of the $J$ sites every $\triangle t$, and that any change of state at any site occur accordingly. The output of a simulation can provide the species composition at each of the $J$ sites after every time interval $\triangle t$.

The full model should be evaluated over many different initial state vectors $N_{0}$; computations for each $N_{0}$ should be repeated to characterize the probabilistic results adequately. The size of the simulated environment has to be suffiently large to render the influence of the border regions (where the ecological stencil is incomplete) negligible. Consequently, the amount of required computing will be very large, and parallelism is necessary to achieve acceptable simulation times. We have had some success implementing the full model on a SIMD (Single Instruction Multiple Data) computer (Maniatty et al. 1992). The environment is simulated by an assemblage of autonomous stochastic automata organized into a two-dimensional cellular automaton. There is a match between a model site's immediate ecological neighborhood (Von Neumann and Moore neighborhoods; see Reggia et al. 1993) and the interconnection network of the parallel computer used to simulate our model. This concordance between the model environment and the parallel computer leads to particularly simple and elegant implementation.

\section{Applications}

The full model might be considered a vector-borne recurrent epidemic with two susceptible populations. "Recurrent" implies that the size of each host population changes because of natality/dispersal and mortality during the course of the epidemic. If we eliminate dispersal and mortality of each host, and eliminate mortality of the pathogen, we have a spatially explicit version of the simple epidemic (see Bartholomew, 1983). The simple epidemic assumes a constant total size of the host population(s) and excludes recovery from disease (no pathogen loss). Parasitism can be interpreted as contact (of random duration) between nearby individuals in our version of the simple epidemic. If we add pathogen loss to the simple epidemic, our model becomes a spatially explicit general epidemic, since infected hosts can recover from disease (e.g. [1]).

By extending the set of states $\xi_{k}$, we could treat recovered hosts as immmune, or less susceptible, to pathogenic infection. One-dimensional epidemic models (diffusions; e.g. Murray and Steward, 1992) suggest that the frequency of the immune class affects the spatial propagation of disease. Our model can ask a similar question in a two- dimensional space.

The spread of disease and the diffusion of culture have similar dynamics (Bartholomew, 1983; Cavalli-Sforza and Feldman, 1981; Giraldeau et al., in press). Knowledgable individuals pass information to "ignorants" through social learning in a manner mathematically analogous to an epidemic. Therefore, each of the spatially explicit epidemic models can be applied to the associated paradigm for cultural diffusion.

Our model represents a closed ecological system. By adding probabilities of immigration from outside sources we can simulate an open system. Immigration obviously has biological significance and may assure a stochastic equilibrium for the spatial process (Kelly, 1979), since extinction would no longer trap the system. An open system would allow us to examine interactions involving dispersal, mortality, and immigration rates. We invite suggestions of biological problems that extend the applications of our model and require simulation experiments for a complete analysis. 


\section{Discussion}

Our paper develops a modeling technique for predicting spatio-temporal properties of a diverse set of ecological and epidemiological interactions. Varying the model's parameters allows simulation of a variety of probabilistic dynamic systems. Just as importantly, varying the size and shape of the ecological stencil should produce a series of new predictions linking local ecological interactions to large- scale community characteristics.

Our analytical comment on quasi-stationary densities of two competing species indicates that a poor competitor can remain common, while a good competitor remains rare, for extended periods. In Lotka-Volterra competitive systems with homogeneous mixing (i.e. no spatial structure) numerical superiority can allow the common species to exclude the rare when both species are regulated more by interspecific competition than by intraspecific competition. The dynamics of our model quantify an interspecific competitive effect, but we do not include explicit self-regulation within the ecological stencil (although global availability of open sites can constrain the growth of either host). As the ecological stencil grows large, our model approaches the homogeneous mixing case; local densities approach global densities. Consequently, the poor competitor's numerical superiority suppresses dispersal and growth of the good competitor. If the ecological stencil is small, however, the good competitor's higher dispersal and lower mortality are effective, since the poor competitor's global abundance does not suppress the good competitor locally. Site by site, the good competitor's abundance will increase and the poor competitor will be excluded ultimately. These results imply that the significance of competitive asymmetries in nature may at times depend on dispersal distances, hence, on the size of the ecological stencil.

The primary goal of our simulations will be specifying predictions concerning the influence of vector-borne disease on community structure and diversity, at an ecological time scale. A number of studies have assumed a longer time scale and asked how evolution or coevolution might govern interaction rates of exploiter-victim systems (e.g. Rosenzweig and Schaffer, 1978; Schaffer and Rosenzweig, 1978). This general framework includes coevolution of host resistance with the virulence of parasites and pathogens (e.g. Marquis and Alexander, 1992).

Straightforward modification of our model will allow us to mimic selection on variation in levels of the model's parameters. We might, for example, introduce two pathogens differing in virulence. Greater virulence might imply increased host mortality $\left(\mu_{h+4}, \mu_{h+6}\right)$ and/or decreased host dispersal $\left(\rho_{h+4}, \rho_{h+6}\right)$. The next step could allow variation in host resistance (decreased host susceptibility $\beta_{h}$ and/or increased host recovery $\mu_{v}$ ). Coevolution between pathogenic virulence and host resistance might best be accomplished by a shorter time tick for the pathogen, since host generation length will ordinarily exceed that of its pathogens. Further analyses could examine the evolution of parasite selectivity when increasing the rate of attack on one host type reduces the parasite's capacity to exploit the other host. The particular advantage of our model will be that we can approach these questions and record the results through spatially explicit population dynamics.

\section{Acknowledgements}

We thank B. Maniatty and M. L. Rosenzweig for comments on the manuscript.

\section{References}

[1] Anderson, R.M. (1991) The Eighth Tansley Lecture. Populations and infectious diseases: ecology or epidemiology? J. Anim. Ecol. 60, 1-50.

[2] Bartholomew, D.J. (1983) Stochastic Models for Social Processes, 3rd Edn. Wiley, New York, USA. Bergelson, J. (1990) Life after death: site pre-emption by the remains of Poa annua. Ecology 71, 2157-65.

[3] Cavalli-Sforza, L.L. and Feldman, M.W. (1981) Cultural Transmission and Evolution: A Quantitative Approach. Princeton University Press, Princeton, USA.

[4] Giraldeau, L.-A., Caraco, T. and Valone, T.J. (In press) Social foraging: individual learning and cultural transmission of innovations. Behav. Ecol. 
[5] Hastings, A. (1990) Spatial heterogeneity and ecological models. Ecol. 71, 426-28. Hochberg, M.E., Hassel, M.P. and May, R.M. (1990) The dynamics of host-parasitoid-pathogen interactions. Am. Nat. 135, 74-94.

[6] Hosford, R.M. (1967) Transmission of plant viruses by dodder. Bot. Rev. 42, 387-406.

[7] Kareiva, P. (1990) Population dynamics in spatially complex environments: theory and data. Phil. Tran. Roy. Soc., London, B, 30, 175-90.

[8] Karmeshu and Pathria, R.K. (1979) Cooperative behaviour in a nonlinear model of diffusion of information. Can. J. Physics 57, 1572-78.

[9] Kelly, C.K. (In press) Resource choice in Cuscuta europaea. Proc. Natl. Acad. Sci., USA.

[10] Kelly, F.P. (1979) Reversibility and Stochastic Networks. Wiley, New York, USA.

[11] Maniatty, W., Sinharoy, B. and Szymanski, B. (1992) Efficiency of data alignment on MasPar. SIGPLAN Notices 28, 48-51.

[12] Marquis, R.J. and Alexander, H.M. (1992) Evolution of resistance and virulence in plant-herbivore and plant- pathogen interactions. Trends Ecol. Evol. 7, 126-29.

[13] Murray, J.D., F.R.S., Stanley, E.A. and Brown, D.L. (1986) On the spatial spread of rabies among foxes. Proc. Roy. Soc., London, B, 229, 111-50.

[14] Murray, J.D. and Steward, W.L. (1992) On the spatial spread of rabies among foxes with immunity. J. Theor. Biol. 156, 327- 48.

[15] Pacala, S.W. and Silander, J.A., Jr. (1985) Neighborhood models of plant population dynamics. I. Singlespecies models of annuals. Am. Nat. 125, 385-411.

[16] Portnoy, S. and Willson, M.F. (1993) Seed dispersal curves: behavior of the tail of the distribution. Evol. Ecol. 7, 25-44.

[17] Power, A.G. (1987) Plant community diversity, herbivore movement, and an insect-transmitted disease of maize. Ecol. 68, 1658- 69.

[18] Power, A.G. (1989) Cropping systems, insect movement, and the spread of insect-transmitted diseases in crops. The Ecology of Agricultural Systems (R. Carrol, P. Rosset and J. Vandermeer, eds.), MacMillan Press, New York, USA.

[19] Reggia, J.A., Armentrout, S.L., Chou, H.-H. and Peng, Y. (1993) Simple systems that exhibit self-directed replication. Science 259, 1282-87.

[20] Rosenzweig, M.L. and Schaffer, W.M. (1978) Homage to the Red Queen. II. Coevolutionary response to the enrichment of exploitation ecosystems. Theor. Pop. Biol. 14, 158-63.

[21] Roughgarden, J.D., Pacala, S.W. and Rummel, J.D. (1984) Strong present-day competition between Anolis lizard populations of St. Maarten (Neth. Antilles). Evolutionary Ecology (B. Shorrocks, ed.), Blackwell, London, UK.

[22] Schaffer, W.M. and Rosenzweig, M.L. (1978) Homage to the Red Queen. II. Coevolution of predators and their victims. Theor. Pop. Biol. 14, 135-57.

[23] Schall, J.J. (In press) Parasite-mediated competition in Anolis lizards. Oecologia.

[24] Schoener, T.W. (1976) Alternatives to Lotka-Volterra competition: models of intermediate complexity. Theor. Pop. Biol. 10, 309-33.

[25] Sharma, C.L., Pathria, R.K. and Karmeshu (1982) Critical behavior of a class of nonlinear models of diffusion of information. Phys. Rev. A 26, 3567-74. 
[26] Shmida, A. and Ellner, S. (1984) Coexistence of plant species with similar niches. Vegetatio 58, 29-55.

[27] Sinharoy, B. and Szymanski, B.K. (1993) Finding optimum wavefront of parallel computation. Proceedings of 26th Hawaii International Conference on System Sciences. IEEE Computer Science Press, New York, USA.

[28] Stephens, D.W. and Krebs, J.R. (1986) Foraging Theory. Princeton University Press, Princeton, USA. Sutherland, J.P. (1974) Multiple stable points in natural communities. Am. Nat. 108, 859-73. 